$\Rightarrow$ DNA REPLICATION

\section{Unwinding maxicircle DNA}

How the replication of mitochondrial DNA (mtDNA) is synchronized with nuclear DNA replication and how the copy number is maintained are largely unclear. However, a new study of trypanosome kinetoplast DNA (kDNA) - mtDNA that consists of minicircles and maxicircles - reveals how maxicircle replication might be regulated (or 'licensed' to initiate DNA replication just once per cell cycle) to control copy number and maintain genomic stability.

Following preliminary studies that identified six candidate helicases in Trypanosoma brucei, Liu et al. show that one of these helicases, TbPIF2, has an integral role in replicating $\mathrm{kDNA}$ maxicircles. To investigate its function in $\mathrm{kDNA}$ replication, the authors used RNA interference (RNAi) to reduce TbPIF2 expression. This resulted in a decrease in cell growth after 6 days and a virtually complete loss of maxicircles after only 1 day of RNAi, but had little effect on minicircles. These observations indicate that TbPIF2 expression is necessary for maxicircle replication.
Consistent with this, overexpression of TbPIF2 resulted in a threefold to sixfold increase in the numbers of maxicircles after 1 day, suggesting that the levels of TbPIF2 correspond to the numbers of maxicircles. TbPIF2 overexpression also affected kDNA segregation during cell division, with some cells having small or no kDNA and others having unsegregated, large kDNA. Furthermore, overexpression of a catalytically inactive TbPIF2 hindered cell growth and reduced maxicircle numbers, which suggests that the helicase

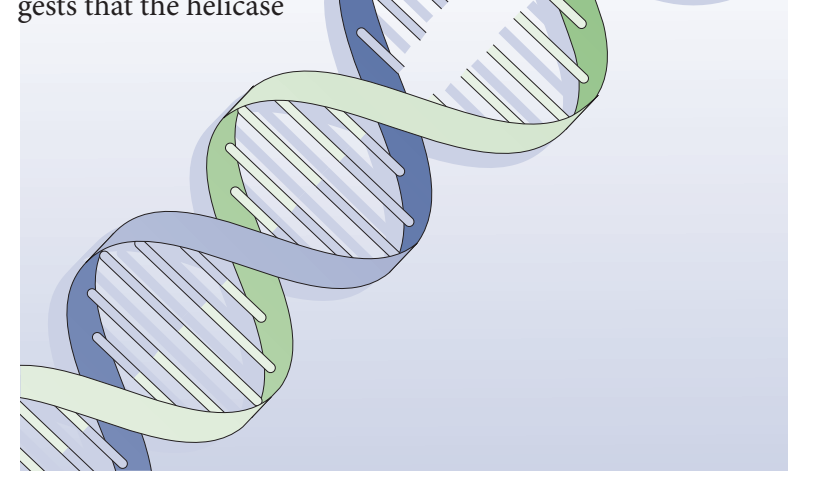

activity of TbPIF2 is an important licensing factor in kDNA replication.

So how is maxicircle copy number controlled? The replication of $\mathrm{kDNA}$ is known be regulated by the mitochondrial proteasome-like protease TbHslVU, which is thought to degrade proteins that control minicircle and maxicircle replication. Indeed, depletion of TbHslVU by RNAi resulted in a fivefold increase in TbPIF2 levels, revealing a possible mechanism for regulating the replication licensing of

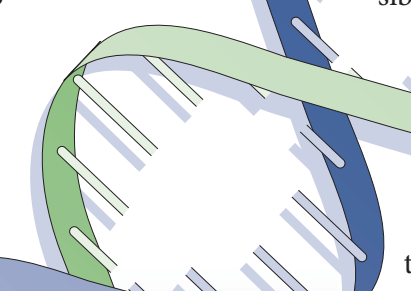
maxicircles, and possibly of mtDNA in general. Taken together, these data provide a molecular mechanism for maxicircle kDNA replication that involves positive regulation by the DNA helicase TbPIF2, which is in turn regulated by the protease TbHslVU. It is not difficult to envisage that helicase turnover could provide a general mechanism for regulating mtDNA copy numbers.

Rachel David

ORIGINAL RESEARCH PAPER Liu, B. et al.

Trypanosomes have six mitochondrial DNA helicases with one controlling kinetoplast maxicircle replication. Mol. Cell 35, 490-501 (2009) 